\title{
Novel micro-optical collector design for a gas chromatographic detector based on atomic emission spectroscopy
}

\author{
Matthias Gruber, MEMBER SPIE \\ Michael Bohling \\ Holger Winkelmann \\ Hans Knuppertz \\ University of Hagen \\ Department of Mathematics and Computer Science \\ Universitätsstr. 27 \\ 58097 Hagen, Germany \\ E-mail: matthias.gruber@fernuni-hagen.de
}

\begin{abstract}
We report a novel micro-optical systems approach for gas chromatographic detection based on plasma excitation of the eluate and subsequent emission spectroscopic evaluation. Specifically, we propose a detector architecture that integrates a microhollow cathode setup and an optical collector system on a common planar microsystems platform. The collector consists of an array of identical imaging systems that surround the microplasma and couple the emitted light side-on into fibers via which it can be fed into a spectrometer. Elliptically shaped reflector profiles ensure nearly aberration-free achromatic imaging and hence a high coupling efficiency. This is confirmed by ray-tracing simulations. An experimental demonstration of the detector module is assembled. The elliptical profiles are milled out of aluminium with diamond tools on an ultraprecision machining center. Experimental tests with a He plasma prove that a higher optical coupling efficieny than with the traditional end-on signal pickup scheme can be achieved. (C) 2010 Society of Photo-Optical Instrumentation Engineers. [DOI: 10.1117/1.3516731]
\end{abstract}

Subject terms: ionization spectroscopy; plasmas; micro-optics; imaging systems; fiber optic applications.

Paper 100602R received Aug. 3, 2010; revised manuscript received Sep. 24, 2010; accepted for publication Oct. 5, 2010; published online Dec. 6, 2010.

\section{Introduction}

Gas chromatography (GC) is a chemical analysis method that is based on the separation and subsequent identification of gaseous material samples. It involves a mobile phase and a stationary phase, the former one consisting of the analyte and a carrier gas, the latter one of a microscopic liquid layer in a thin tubing, the so-called GC column. After injection of the mobile phase into the column, different analyte compounds interact differently with the liquid phase, resulting in specific retention times elapsing before the compounds are eluted from the column and detected. Due to its high sensitivity, GC has become an important analytical tool for biochemical, environmental, and forensic applications. ${ }^{1}$

Our focus lies on the detection step at the end of the GC column. The ideal detector should be highly sensitive, respond rapidly, and have a large dynamic range. An additional beneficial feature is universality, i.e., the detector responds to any atomic or molecular species and generates a unique, easily measurable signal on their presence. Detectors based on emission spectroscopy are capable of fulfilling these requirements reasonably well. The basic idea is to excite the eluate so that its compounds will emit their characteristic atomic or molecular line spectra, which can then be detected and identified with an optical spectrometer (cf. Fig. 1).

Plasmas generated by electrical discharges are highly suitable for the task of energetic excitation. Since an early demonstration $^{2}$ in 1965 , this technological approach has been implemented successfully with a variety of electrical discharge types. More recently, the focus has shifted toward microplasmas and miniaturized GC detector architectures

0091-3286/2010/\$25.00 @ 2010 SPIE that fit into the concept of microsystems integration ("labon-a-chip") and are compatible with established microfabrication techniques. ${ }^{3,4}$ Expected benefits of the lab-on-a-chip approach are mobility, lower analyte and process gas consumption, and reduced analysis time.

This paper aims at a particular type of microplasmas, those generated by so-called micro-hollow cathode discharges ${ }^{5}$ (MHCDs), and focuses on a problem that has received only minor attention in prior investigations: the efficient collection of the radiation emitted by the excited eluate and the optical coupling into a portable fiber optic spectrometer. We propose a novel architecture for an emission-spectroscopic GC detector that combines the functions of microplasma generation and optical collection on a common microtechnological platform.

After a brief description of the physical characteristics of MHCDs in the following section, we present the general scheme of the proposed detector architecture in Sec. 3. Section 4 discusses its key optical components, elliptically shaped reflectors. Ray-tracing simulations in Sec. 5 confirm the viability of our approach theoretically. Sections 6 and 7 report the fabrication of an experimental demonstrator and basic functional tests, respectively.

\section{MHCDs}

MHCDs are derivatives of a classical electrical discharge archetype that is generated by means of a macroscopic tubular-shaped cathode, as depicted in Fig. 2(a). This electrode geometry leads to a voltage drop (cathode fall) in the radial direction and hence to a discharge between the circular cathode and a virtual anode on the axis of the hollow cathode. Electrons repeatedly bounce back and forth in this potential field (pendulum effect) and experience a large number of 


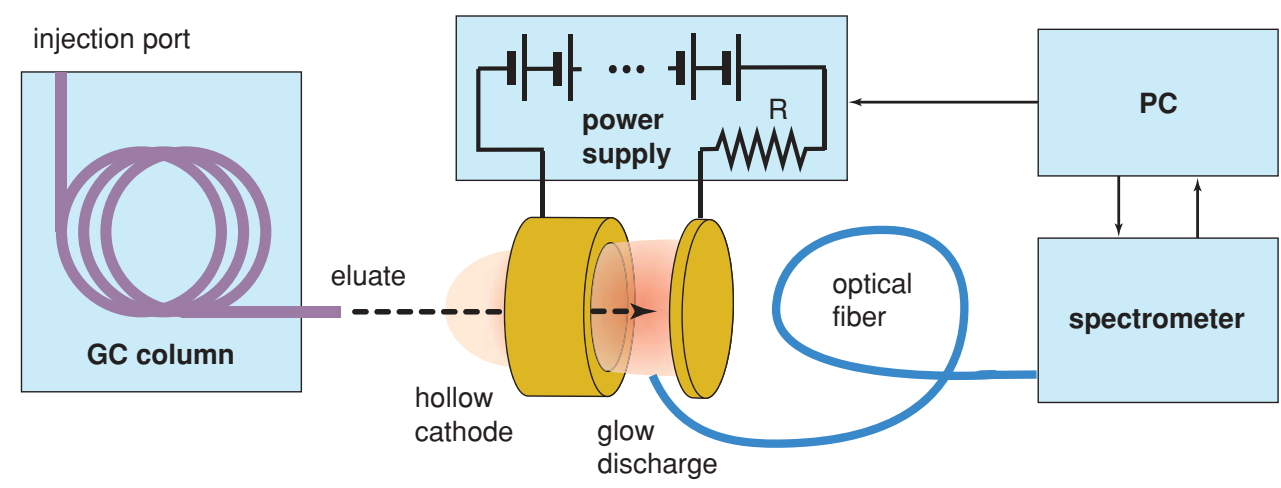

Fig. 1 GC detection principle based on plasma excitation and atomic emission spectroscopy.

collisions, which is a major cause for the high ionization and excitation efficiency of hollow cathode discharges. ${ }^{6}$ The negative glow, which acts as the optical signal source in an application such as a GC detector, can thus reach a high radiance.

Interestingly, it is possible to modify the classical electrode arrangement of Fig. 2(a) significantly without sacrificing these desirable electrical and optical characteristics. Investigations by Schoenbach et al. ${ }^{7}$ showed that this holds even for miniaturized architectures that are fabricated with planar techniques, as depicted in Fig. 2(b). The setup consists of an electrically insulating wafer with metal sheet electrodes on both sides and a tiny hole through these three layers. If the hole diameter is sufficiently small (typical range is 100 to $300 \mu \mathrm{m})$ a MHCD will arise and the negative glow will be located inside the hole around the center axis.

The approach of Fig. 2(b) has several interesting features. The tubular nature of all three layers (including the anode) makes it very easy to connect a MHCD setup to a GC column; if properly positioned, the eluate can flow through the hole almost without obstruction. Second, due to the symmetry of the setup, discharge processes must be invariant with respect to the polarity of the electrodes, i.e., the anode and cathode can be interchanged. This means that the setup can also be operated with ac voltage; in this case, it is recommendable to use a high frequency (preferably rf in the megahertz range) because this helps to reduce sputtering effects and thus degradation of the electrodes. Another favorable feature is related to a similarity law that governs electrical discharges. Refered to as Paschen's law, it states that the breakdown voltage that is necessary for plasma initiation depends on the product of gas pressure $p$ and electrode distance $d$, the minimum voltage is obtained for a specific $p d$ product. To reach this optimum operating point, macroscopic discharge chambers must usually be evacuated; with an MHCD setup such as is Fig. 2(b), however, it can be reached under atmospheric pressure. This

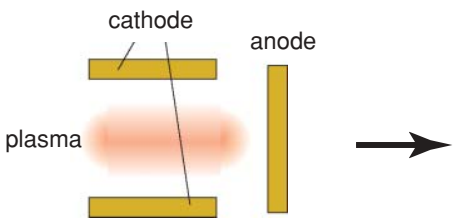

(a)



(b)
Fig. 2 Cross section of (a) a classical hollow cathode and (b) a MHCD setup. constitutes a crucial advantage for mobile or lab-on-a-chip applications.

Practical tests with MHCD devices have confirmed their suitability as emission spectroscopic GC detectors. ${ }^{4}$ These tests, however, focused almost exclusively on the physical characteristics of the microplasmas. For the optical coupling between microplasma and spectrometer simple ad hoc solutions were employed, usually end-on* imaging onto the fiber end with a discrete single lens. This is where our effort sets in. We integrate the MHCD device and a micro-optical collection and coupling system on a common platform.

\section{Novel System Architecture}

Fundamentally, the proposed detector architecture originates from the idea to combine the inherently planar MHCD approach with a planar optical integration concept, specifically with planar-integrated free-space optics ${ }^{8}$ (PIFSO). One of the characteristic features of PIFSO is zigzag-type signal propagation inside a transparent wafer. Applying the same principle here leads to an architectural scheme with a side-on signal pickup, ${ }^{9}$ as illustrated in Fig. 3. One can recognize a MHCD setup consisting of metal electrodes and a small central hole on a transparent wafer. This inlay part is sandwiched between a top plate and a bottom plate, which serve both as plane mirrors and as mechanical support, also for the optical fibers that connect the system to a spectrometer and that are arranged in a star-like fashion around the center.

It is assumed that a large fraction of the characteristic radiation that is emitted from the microplasma inside the central hole will couple into the surrounding transparent wafer and propagate in the outward direction, thereby undergoing several reflections at the wafer surfaces and the top and bottom plates. Light rays that eventually hit the core area of a fiber at an angle smaller than its acceptance angle will be coupled in and contribute to the optical signal that is transmitted to the spectrometer. In other words, the optical function of the setup in the $x-z$ cross section is that of a (highly multimode) planar waveguide.

In the orthogonal $x-y$ cross section, the microplasma-tofiber coupling is achieved by optical imaging operations. By splitting the collector design into sectors with identical optical functions it is possible both to cover a large solid angle, in particular the full $2 \pi$ range of the polar angle, and to

\footnotetext{
*In accordance with the terminology in the literature, the terms "end-on" and "side-on" refer to observation directions parallel and perpendicular to the symmetry axis of the MHCD, respectively.
} 


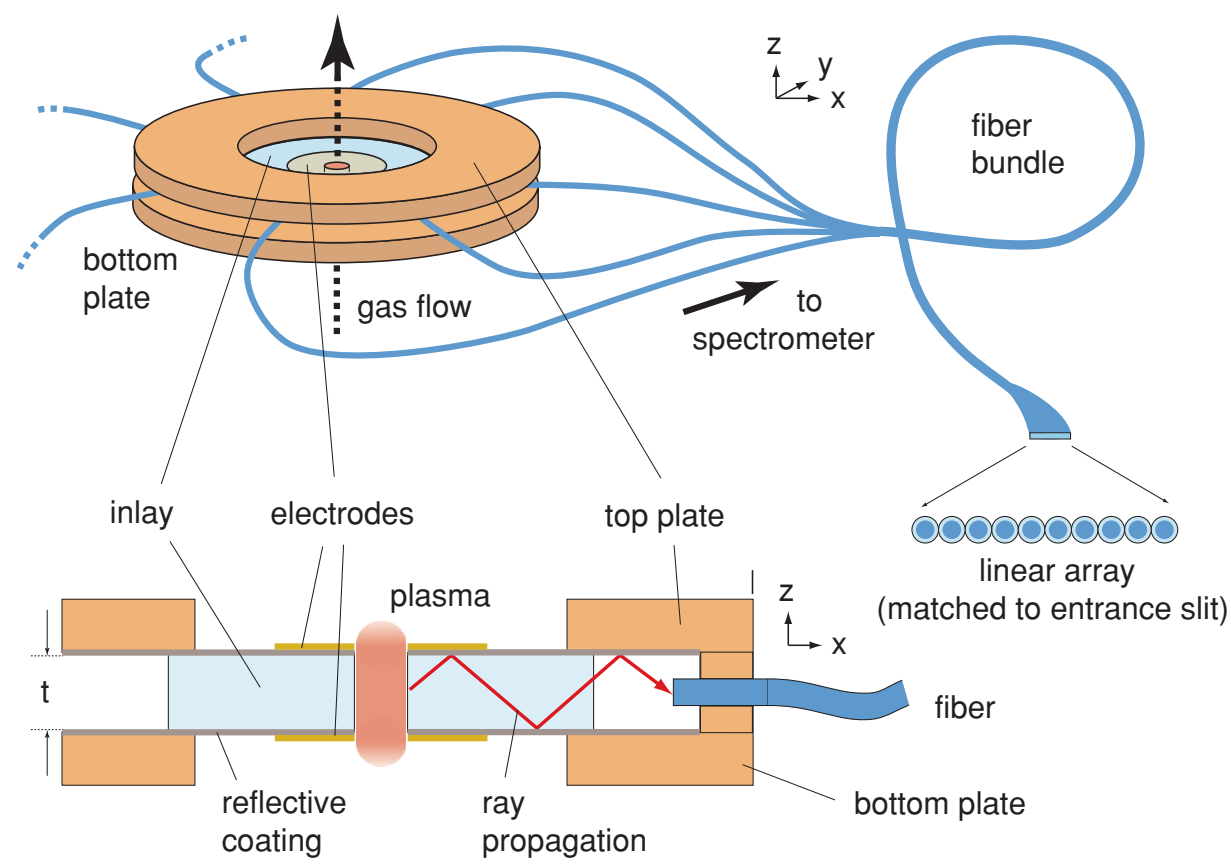

Fig. 3 Novel planar system architecture with integrated MHCD setup and collector optics.

adapt the numerical aperture of an individual imaging system to that of the optical fibers. A significantly larger fraction of the total emitted radiation than with the traditional singlefiber approach can thus be captured; the improvement factor approximately equals the number of fibers used. However, the high angular coverage will lead to a high overall system efficiency only if the collected radiation is also efficiently coupled into the spectrometer. To this end, we propose to arrange the spectrometer-sided fiber ends in a linear array that matches the geometry of the entrance slit.

For the microplasma-to-fiber imaging task, dioptric or catoptric solutions can be devised. Figure 4 exemplarily depicts the first kind, which was initially favored. Here, each sector of the transparent wafer has a curved edge profile that acts as a focusing lens. If this profile is shaped as a Cartesian oval, ${ }^{10}$ then the imaging of the center of the micro-hollow cathode (MHC) hole onto the fiber core will even be stigmatic for any numerical aperture, which promises low coupling loss. However, a refractive optical systems approach is sensitive to material dispersion and will display chromatic aberrations. The plasma-to-fiber coupling efficiency $\eta$ will therefore decrease for wavelengths different from the design

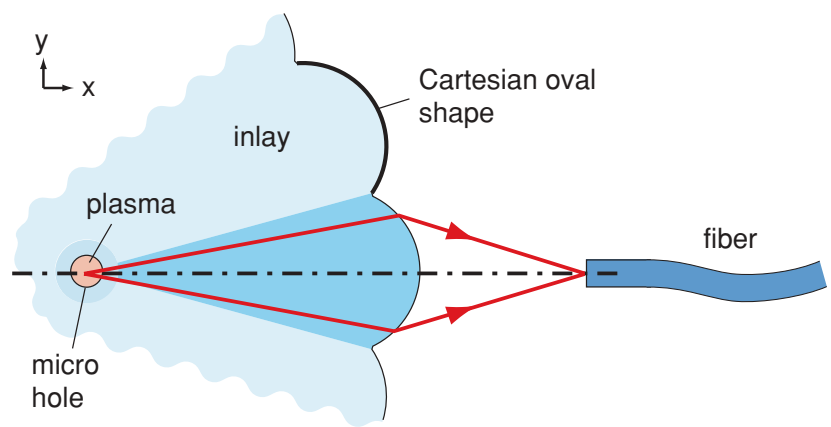

Fig. 4 Dioptric approach for the plasma-to-fiber imaging task. wavelength, and the effect can be significant for broadband applications, as in our case. Ray-tracing simulations indicate that with fused silica as wafer material, $\eta$ typically drops to less than half of its maximum value assuming ${ }^{11}$ a design wavelength of $\lambda=550 \mathrm{~nm}$ and operation at $\lambda=400 \mathrm{~nm}$. This is why dioptric approaches were eventually discarded in favor of a inherently achromatic catoptric alternative.

A particular challenge of reflective imaging systems is due to the fact that object and image domains usually overlap. This can easily cause sterical problems. A sophisticated optical design is necessary to avoid beam obstructions by system components. For the GC detector, we developed an architecture consisting of a circular array of identical elliptically shaped reflector profiles, as depicted in Fig. 5. The

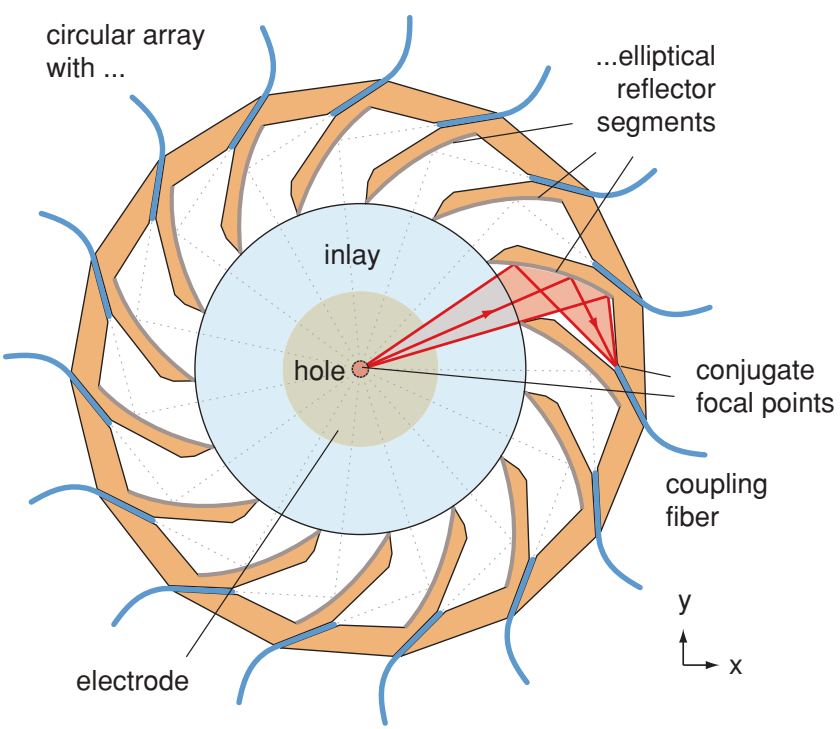

Fig. 5 Catoptric approach for the plasma-to-fiber imaging task. 
profiles are curved in such a way that one focal point of each ellipse coincides with the center of the MHC hole, whereas the end of one of the spectrometer fibers is positioned in each second focal point. In this way, one can take advantage of the fact that the two focal points of an ellipse are conjugate points in the technical-optical sense and that the imaging is strictly stigmatic.

Furthermore, the design approach of Fig. 5 makes it possible to cover the polar angle with a fill factor of nearly $100 \%$. The transparent wafer with the MHCD structure was designed as a flat cylindrical inlay into the reflector array. Due to its circular symmetry, the outer edge of the inlay has no technical-optical effect since all rays emerging from the center will intersect the edge perpendicularly. Material dispersion effects are therefore irrelevant. The choice of suitable geometric parameters for the elliptic collectors are discussed next.

\section{Elliptical Reflector Design}

For the design of the elliptical profiles there are essentially three adjustable parameters: (1) the overall size, (2) the eccentricity, and (3) the segment of the elliptical curve that is actually used. General design goals are high coupling efficiency, good manufacturability, and convenient experimental handling. Since a change of the overall size is simply a scaling, it renders a geometrically similar system with identical ray-optical properties. Parameter 1 therefore has no direct influence on the coupling efficiency, it affects only manufacturability and handling. In our particular case, the upper limit for the overall size was determined by the working range of the machining center, the lower limit by the dimensions of the substrate base material that we intended to use for the inlay part.

The situation is different for parameters 2 and 3 . To recognize their influence more clearly we first deduce more specific system requirements from the general design goals. These are

1. sufficiently large cavity for the inlay

2. no beam obstruction

3. a $100 \%$ angular fill factor

4. high imaging quality

Requirement 1 is obviously essential to realize the design approach, as outlined in Fig. 5. It implies that the two focal points $F_{1}$ and $F_{2}$ of the ellipse must have a distance that is larger than the inlay diameter. Together with the requirement that the total collector size should not be orders of magnitude larger than the inlay it follows that the eccentricity of the ellipse must be rather large. The same conclusion can been drawn from requirement 2. As one can see from Fig. 6 no beam obstruction means in particular that the light ray $P_{1} F_{2}$ must not intersect the edge of the circular inlay again after reflection at point $P_{1}$. This requires an obtuse reflection angle $\beta$; in the specific example of Fig. 6, the demand $\beta>90 \mathrm{deg}$ is equivalent to $e=c / a>\sqrt{2}$ for the numerical eccentricity. Requirements 2 and 3 imply that a free area remains between the circular inlay area, the beam area, and the fiber area for incorporating a neighboring reflector profile; at point $Q_{1}$ this profile is just in contact with the ray $F_{1} P_{2}$. Furthermore, the fiber channel must not intersect the lower part of the neighboring reflector and should be directed outward; i.e., $\gamma$ should be an acute angle. For this to be the case, it is not only

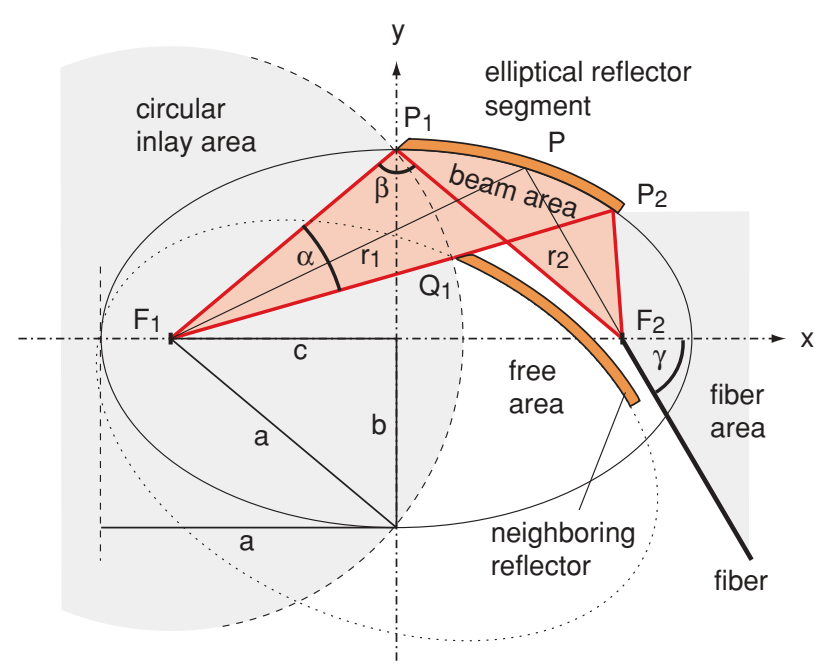

Fig. 6 Design considerations for elliptically shaped reflector profiles.

necessary to have a rather large eccentricity but also to use a segment of the ellipse in the vicinity of the minor axis (the $y$ direction in Fig. 6). To leave a sufficiently large inlay cavity we recommend $P_{1}$ to be located on the $y$ axis and $P_{2}$ in the halfplane of $F_{2}$ but only as far away from the minor axis that the inclination of ray $P_{2} F_{2}$, and hence, the inclination of the fiber axis also still satisfies the aforementioned condition.

Requirement 4 is an issue for the optical system design because the object (microplasma in the hole) has a small but finite extension but only the two focal points of an ellipse are imaged strictly stigmatically onto each other. For an imaging analysis of extrafocal points, it is convenient to look $^{12}$ at the transverse magnification $m$. This quantity can be defined along every principal ray path $F_{1} P F_{2}$, for small ray pencils the paraxial relation $m=r_{2} / r_{1}$ is valid. The ratio $r_{2} / r_{1}$, however, is continuously changing across the elliptical mirror, $m$ is larger for pencils that hit the reflector near $P_{1}$ than for those hitting it near $P_{2}$. At the fiber end in $F_{2}$, images of different sizes will therefore be superimposed, which will usually cause coupling loss unless the fiber core is significantly overdimensioned. This problem is system-immanent but can be alleviated by avoiding too large variations of the ratio $r_{2} / r_{1}$. Since they are directly linked with the eccentricity it is necessary that this parameter is bounded above.

On a more theoretical level the phenomenon of varying magnification has to do with the Abbe sine condition. From the definition ${ }^{13}$

ny $\sin U=n^{\prime} y^{\prime} \sin U^{\prime}$,

with $n, y$, and $U$ as the refractive index, the object/image height, and the aperture angle, respectively, the condition

$m=\frac{y^{\prime}}{y}=\frac{n \sin U}{n^{\prime} \sin U^{\prime}}=$ const,

can be deduced, i.e., the magnification must be identical for different aperture zones of an imaging system. This is obviously not the case for the elliptical reflectors and the offence against the sine condition (OSC) increases with increasing eccentricity. Since the imaging from $F_{1}$ to $F_{2}$ is free of spherical aberration, the OSC indicates how much the plasma-to-fiber coupling operation will be affected by coma. 


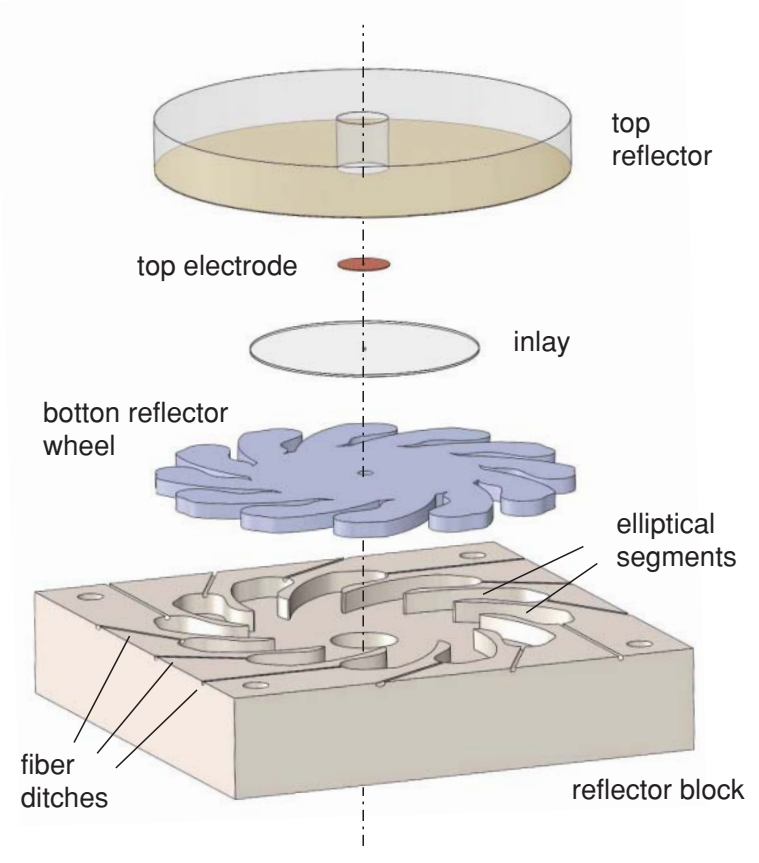

Fig. 7 CAD model of a GC detector module with catoptric collector optics in an exploded view.

The preceding considerations do not lead to just a single solution but rather to a finite "allowed region" in the parameter space. It is roughly marked by the following intervals:

total size: $\quad 20 \mathrm{~mm}<2 a<60 \mathrm{~mm}$, eccentricity: $0.71<e<0.85$,

segment: $10<\alpha<36 \mathrm{deg}$.

What remains to be discussed now is the $z$ extension of the optical system, i.e., thickness $t$ in Fig. 3. On the one hand, with increasing $t$, the size of the light source (lenght of the plasma filament) will increase so that under the assumption of constant radiance, the total emission power will also increase and more light will couple into the inlay. On the other hand, this additional power is practically useless because it cannot be coupled into the fiber. Assuming waveguide-type propagation in the $x-z$ cross section, mode mixing leads to a nearly homogeneous intensity distribution at the outer end of the inaly. Therefore, the inlay-to-fiber coupling efficiency can be assumed to be roughly proportional to the ratio of fiber core diameter and thickness $t$; this ratio decreases as the total power in the inlay increases. As long as the inlay is thicker than the fiber core, one can thus assume that the system efficiency is nearly independent of $t$.

\section{Ray-Tracing Simulations}

For further investigations and also for a subsequent practical realization, the following specific parameter set was chosen for the elliptical reflectors:

$$
\begin{aligned}
& a=12.5 \mathrm{~mm}, \\
& b=8.125 \mathrm{~mm}, \\
& e=0.76, \\
& \alpha=30 \mathrm{deg}, \\
& t=0.5 \mathrm{~mm} .
\end{aligned}
$$

This choice was more or less arbitrary within the "allowed region." It leads to a collector system as in Fig. 5 but with 12 identical sectors. For the diameter of the microhole, a typical value of $d_{h}=200 \mu \mathrm{m}$, as reported in Refs. 4 through 7 , was adopted. Finally, core diameter and numerical aperture (NA) of the coupling fibers were set to $200 \mu \mathrm{m}$ and 0.48 , respectively, matching our experimental settings. The overdimensioning of the fiber NA has practical reasons: We want to be able to capture all light even if a fiber is slighly misaligned.

Based on these data, an assembly of the whole detector module was developed and cast into a CAD model. It is shown in Fig. 7 in an exploded view and is discussed later. The assembly, which obviously implements an optical collector as proposed, was ported into ZEMAX to conduct ray-tracing experiments. It was necessary to operate the ZEMAX software in the nonsequential mode because only this approach permits different rays to have a different number of interactions with system interfaces, as is the case in a planar waveguide. The microplasma was modeled as a homogeneous isotropic volume light source, i.e., rays are emitted from random points within the cylindrical hole into random directions. Typically, a total number of $10^{5}$ to $10^{6}$ rays were traced. Ray path plots such as the one in Fig. 8(a) show qualitatively that the focusing and fiber coupling work as expected. Figure 8(b) depicts the irradiance distribution in a window across an outer focal region; one can recognize a striplike focus, as expected. A quantitative evaluation revealed that about $60 \%$ of all rays emitted from the cylindrical source couple into the inlay and remain utilizable. In the outer focal regions, about $8.8 \%$ of

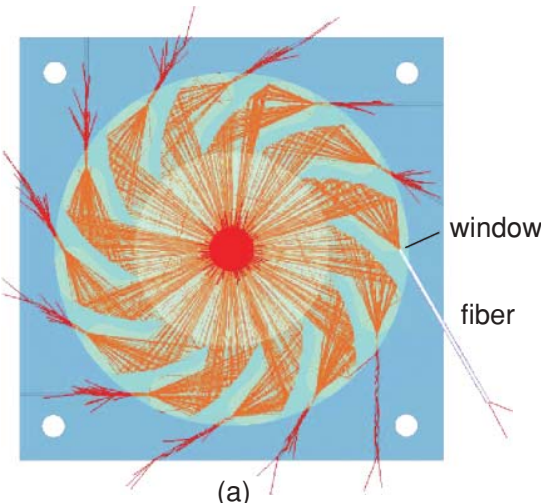

(a)

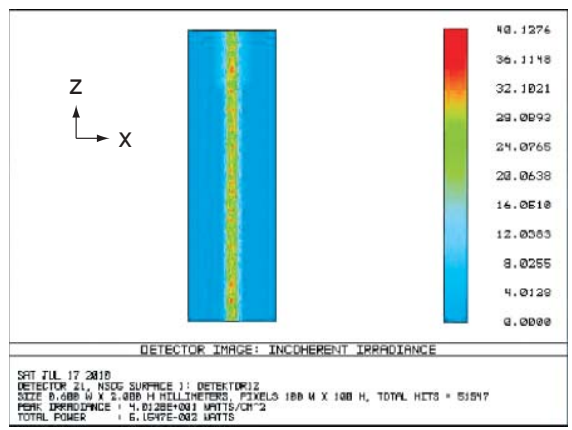

(b)

Fig. 8 Ray-tracing simulations of the catoptric collector model: (a) top view with exemplary ray paths, and (b) irradiance plot across window. 

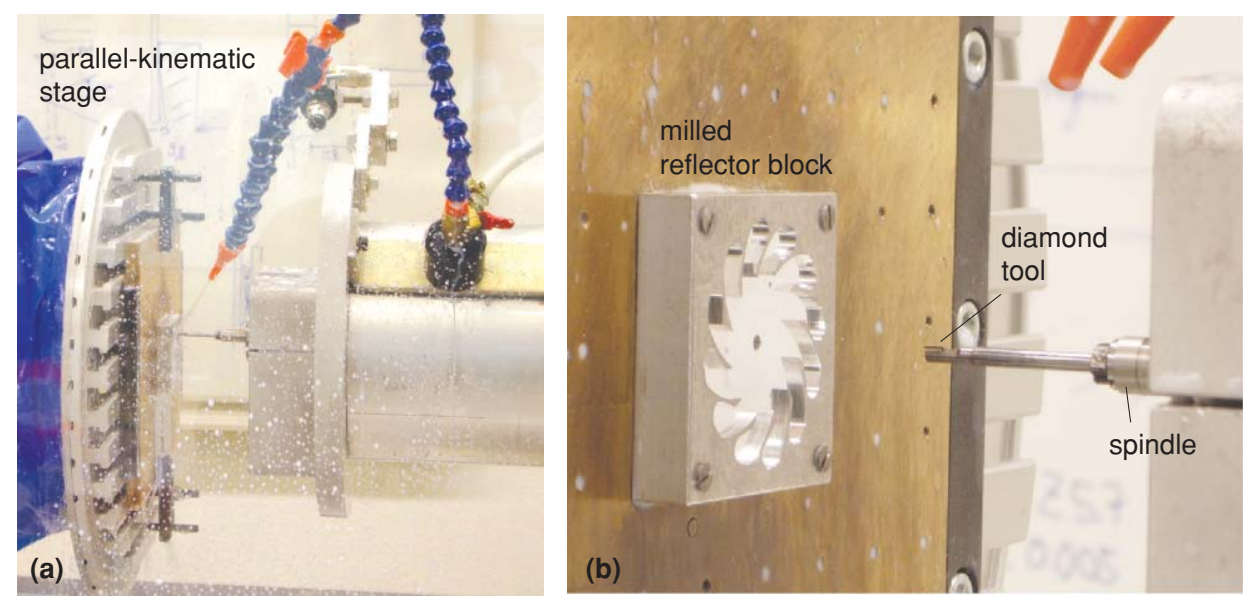

Fig. 9 Fabrication of the elliptical reflector.

the incoming power is typically coupled into the respective fiber.

\section{Fabrication}

The particular assembly of Fig. 7 relates to do the envisaged fabrication technique for the collector optics. We opted for (ultra) precision machining, specifically milling, because today such mechanical approaches enable one to realize truly 3-D structures with optical-quality surfaces. In addition, the components can also take over electrical functions, and a high reflectivity of above $90 \%$ over nearly the entire relevant wavelength range can be obtained if a suitable metal alloy is used as the base material. We experimented with $\mathrm{AlMg}_{3}$, which features a very high resistance to corrosion so that its reflectivity does not degrade, and with $\mathrm{AlZn}_{5} \mathrm{Mg}_{3} \mathrm{Mn}$, which can be polished particularly well. A negative side effect of a machining process is that comparatively large forces are exerted onto the workpiece. To avoid the risk of deforming the delicate elliptical reflector walls we decided to shape the whole system out of a solid material block (cf. Fig. 7), which ensures high stiffness. However, since it is difficult with this approach to obtain optical-grade smoothness for both the elliptical edge profiles and the plane bottom surface we adopt a two-component strategy. In the milled block, we exclusively concentrate on the edge profile quality. The plane bottom mirror is designed as a separate component with an "inverted" elliptical edge profile. This reflector wheel can conveniently be surface-polished before it is inserted into the bottom block; it also serves as the bottom electrode. To be able to accommodate variations of the inlay thickness $t$ the wheel can be adjusted in height by means of three set screws that rest in threads in the bottom block.

As for the transparent inlay part, our design goal was to be able to use low-cost off-the-shelf base products. This led us to microscopic round-shaped coverslips. They require only a finishing polish of the circular edge profile and a small perforation in the center. For the latter task, two technological approaches were considered: mechanical drilling and ablation with femtosecond laser pulses. The cover plate of the system must be electrically insulating to avoid a short circuit between the two MHCD electrodes. This is why the design of Fig. 7 makes use of a dielectric top mirror. A central hole provides access to the small top electrode and serves as an outlet for the probe gas.

The milling of the wheel and the elliptical reflector block was carried out on a parallel-kinematic machining center using cemented-carbide and monocrystalline diamond tools. As one can see from Fig. 9(a), we employed a "wet" process, i.e., an aqueous cooling lubricant was sprayed onto the workpiece. Figure 9(b) depicts an intermediate fabrication stage with finished elliptical profiles. In a subsequent step, the ditches for the optical coupling fibers (cf. Fig. 7) were milled. For this task we used a 0.5 -mm-diam micromilling tool that matches the coating diameter of the fibers. The depth of the ditches is also $0.5 \mathrm{~mm}$, which means that the fibers will just be buried. This can be seen in Fig. 10, which zooms into the focal region of a reflector segment in a partly assembled setup. To prepare the inlay we tested coverslips made of BK7 and fused silica, as well as mechanical and laser-based perforation. We found it possible to obtain operational devices with each of the four alternatives; however, laser ablation provides better yield and smoother hole walls. Our hole diameters range between 0.2 and $0.4 \mathrm{~mm}$.

\section{Functional Tests}

With the tailored components as described above basic functional tests were carried out first. Figure 11 shows a MHCD inlay in a makeshift setup with electrodes made of adhesive copper foil. The purpose of this experiment was to test



Fig. 10 Partially assembled collector module showing a coupling fiber in the second focal point of an elliptical reflector. 


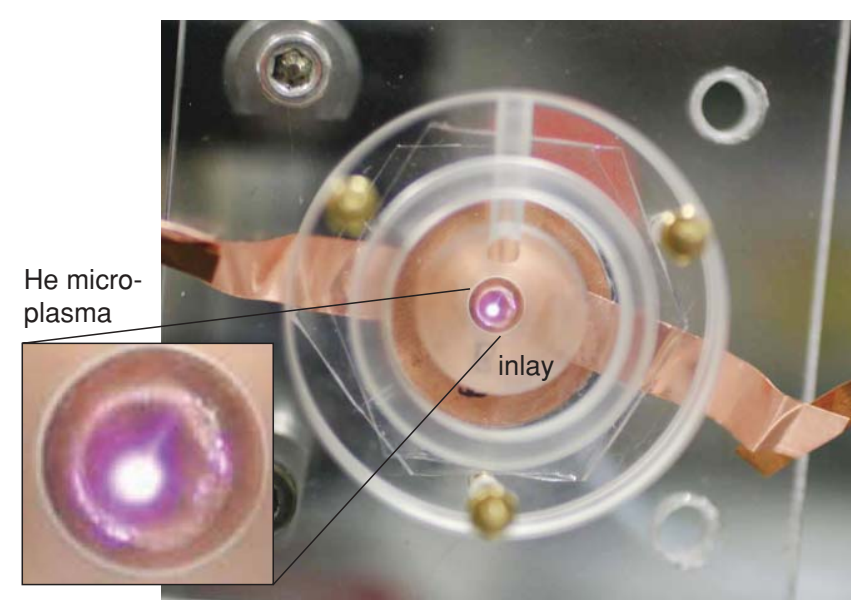

Fig. 11 Trial ignition of a MHCD with the inlay in a He atmosphere.

whether a microplasma can be generated with the device. This is obviously the case, as one can see from the bright pink-colored light emission that is characteristic in a He atmosphere.

Then the complete experimental demonstrator was assembled and connected to a high-voltage power supply (Fig. 12). Tubes for the gas inlet and outlet were plugged onto fittings on the top mirror and bottom block. Again He was used as the test gas and was blown through the MHCD structure at a constant flow rate. After switching the voltage on, the microplasma reliably ignited. Since it is hidden inside the experimental apparatus, it is barely visible from outside in this experiment; however, the coupling into the fibers by the collector optics is efficient enough that the characteristic pink-colored light can now easily be observed with the naked eye at the fiber ends (see enlarged detail in Fig. 12).
Measurements with an optical power meter yielded values of the order of $20 \mathrm{nW}$ per fiber, and with a standard deviation of $3.98 \mathrm{nW}$ in power variation across fibers a satisfactory uniformity.

For comparison with the traditional approach, a reference measurement with an end-on signal pickup scheme was made. For this purpose, the tube at the gas outlet was removed and a single fiber end was inserted through the fitting in axial direction, brought close to the plasma, and adjusted to maximum power, realizing sort of an optical plasma-to-fiber butt-coupling. Note that measurements with this coupling scheme must be carried out quickly because the fiber cannot withstand the high temperature of an atmospheric pressure microplasma (which can exceed $1000 \mathrm{~K}$ ) for a long time. It was found that the measured output power level is about a factor of 2 higher than the level from a single side-on coupled collector fiber; this can be expected since no absorption or scattering loss occurs. However, the reference power level is significantly lower than the cumulative power of all 12 fibers of the demonstrator module (roughly a factor of 6 ). This result experimentally confirmes the usefulness of our GC detector concept.

For the fiber-to-spectrometer coupling a makeshift connector, as shown in Fig. 13 was customized to fit into the SMA 905 jack of a portable Ocean Optics device. In the customized ferrule, the 12 fiber ends are aligned in a linear array. Measurements of the signal levels detected by the spectrometer show that more power can be coupled in than with a standard single-fiber connector. However, with a factor of about 3, the increase is not as large as the cumulative output from the linear array would suggest (factor of 6). We assume that this has to do with the limited clear height of the entrance slit; in our spectrometer, this height appeared to be significantly smaller than the fiber array extension.

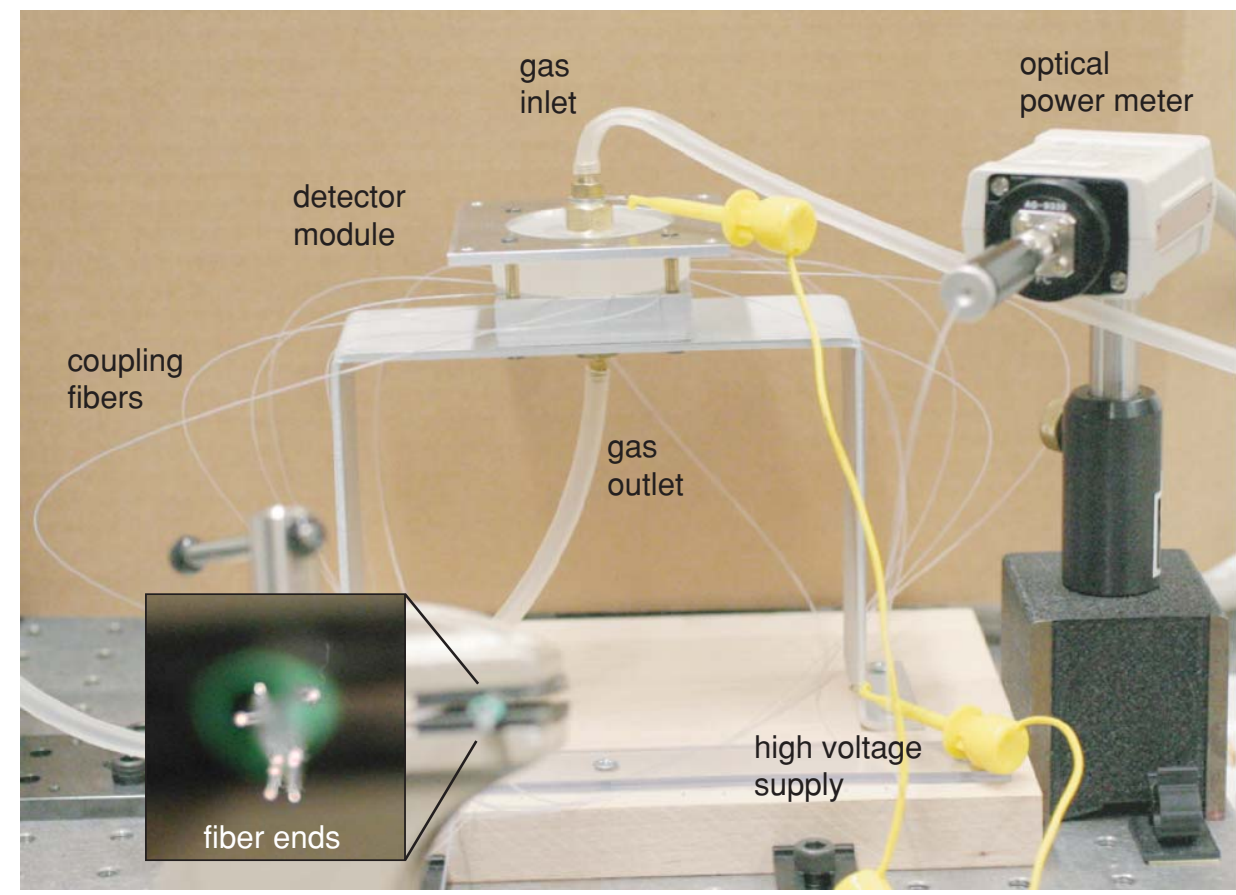

Fig. 12 Functional testing of a fully assembled GC detector module. 


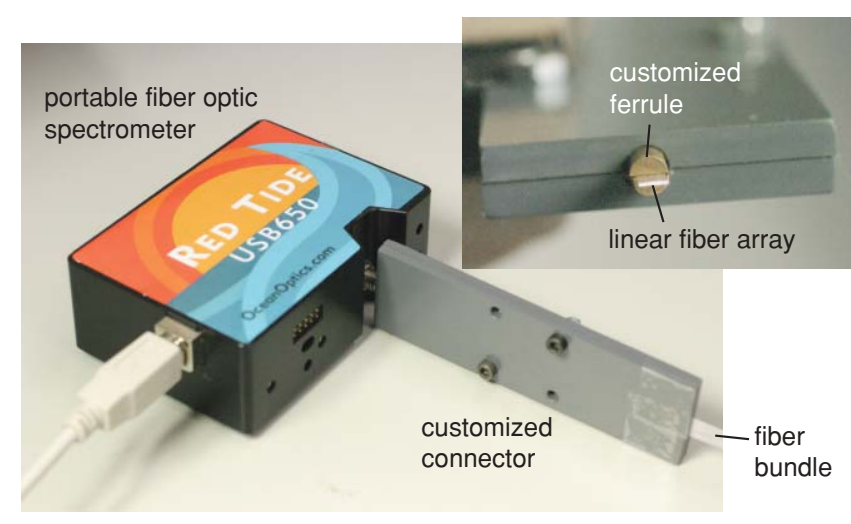

Fig. 13 Customized connector for the fiber-to-spectrometer coupling.

\section{Conclusion}

Although the preceding results mark only a first snapshot in ongoing experimental investigations with a fully assembled detector module, there can be no doubt that they prove the fundamental viability of the proposed systems approach. With the side-on access to the optical signal source and by use of multiple fibers in a matched spatial arrangement it is possible to separate the direction of the sample gas stream from the direction of observation and to maximize the intensity level of the optical signal that can actually be coupled into the spectrometer. Our systems approach is compatible with planar technologies and enables us to integrate a MHCD structure and a micro-optical collector system on a common platform to obtain a compact GC detector module. The sophisticated free-space optical system design consisting of elliptically shaped reflectors is crucial for its high optical coupling efficiency. These reflectors enable an achromatic and nearly aberration-free imaging of the microplasma, i.e., the optical signal source onto the end faces of the coupling fibers. The experimental behavior and computer simulations of the collector system are in good agreement.

\section{Acknowledgments}

Fruitful discussions with Joachim Franzke, Richard Heming, and Cordula Meyer of the Institute for Analytical Sciences (ISAS) in Dortmund, Germany are gratefully acknowledged. We thank the Fachgebiet Technische Optik of the Ilmenau University of Technology, in particular Mr. Roman Kleindienst and Prof. Dr. Stefan Sinzinger for their assistance in laser drilling. This work was funded by Deutsche Forschungsgemeinschaft (DFG) of Germany (grant GR 3343/2-1).

\section{References}

1. R. L. Grob and E. F. Barry, Modern Practice of Gas Chromatography, 4th ed., Wiley, Hoboken, NJ (2004).

2. A. J. McCormack, S. C. Tong, and W. D. Cook, "Sensitive selective gas chromatography detector based on emission spectrometry of organic compunds," Anal. Chem. 37, 1470-1476 (1965).

3. A. Manz, N. Graber, and H. M. Widmer, "Miniaturized total chemical analysis systems: a novel concept for chemical sensing," Sens. Actuat. B 1, 244-248 (1990).

4. M. Miclea, M. Okruss, K. Kunze, N. Ahlmann, and J. Franzke, "Microplasma-based atomic emission detectors for gas chromatography," Anal. Bioanal. Chem. 388, 1565-1572 (2007).

5. M. Miclea, K. Kunze, U. Heitmann, S. Florek, J. Franzke, and K. Niemax, "Diagnostics and applications of the microhollow cathode discharge as an analytical plasma," J. Phys. D Appl. Phys. 38, 17091715 (2005).
6. G. Schaefer and K. H. Schoenbach, "Basic mechanisms contributing to the hollow cathode effect," Chap. 2 in Physics and Applications of Pseudosparks, M. Gundersen and G. Schaefer, Eds., pp. 55-76, Plenum Press, New York (1990).

7. K. H. Schoenbach, A. El-Habachi, W. Shi, and M. Ciocca, "Highpressure hollow cathode discharges," Plas. Sourc. Sci. Technol. 6, 468477 (1997).

8. M. Gruber and J. Jahns, "Planar-integrated free-space optics-from components to systems," Chap. 13. in Microoptics-From Technology to Applications, J. Jahns, K.-H. Brenner, Eds., pp. 225-252, Springer, New York (2004)

9. J. Franzke, M. Gruber, and R. Heming, Plasmagenerator für die spektroskopische Gasanalytik 2009, German Patent No. 10, 2006, 047. 047.

10. E. Hecht and A. Zajac, Optics, 4th ed., Addison Wesley Longman, Amsterdam (2003).

11. M. Bohling, M. Gruber, J. Franzke, and R. Heming, "Evolution of a microplasma chamber and an optical collector into a planarintegrated smart sensor for emission-spectroscopic gas analytics," in Proc. Smart Systems Integration 2009, Mesago Messe Frankfurt (cf. http://www.mesago.de/de/SSI/Der_Kongress/Tagungsband/index.htm) Brussels, Belgium (2009)

12. H. Rehn, "Optical properties of elliptical reflectors," Opt. Eng. 43, $1480-1488$ (2004).

13. H. Gross, "Aberrations," Chap. 11 in Handbook of Optics I, H. Gross, Ed., pp. 485-522, Wiley-VCH, Weinheim (2005).

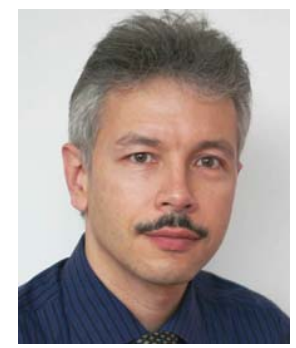

Matthias Gruber received his Diplom degree in physics from the University of Erlangen, Germany, in 1991 and his PhD degree in electrical engineering from the University of Hagen, Germany, in 2003. He is currently an assistant professor of optical microsystems with the Department of Mathematics and Computer Science at the University of Hagen. His research interests include optical information processing, micro-optics, (optical) sensors and actuators, and microelectromechanical systems (MEMS). Dr. Gruber is a member of SPIE, OSA, and EOS.



Michael Bohling received his Diplom degree in applied physics from the University of Applied Sciences in Emden, Germany, in 1988, and his MSc degree in photonics from the University of Hagen, Germany, in 2007. Between 1990 and 2003 he was with several laser material processing companies, eventually in the position of CEO. $\mathrm{He}$ is currently a research assistant with the University of Hagen, Germany.

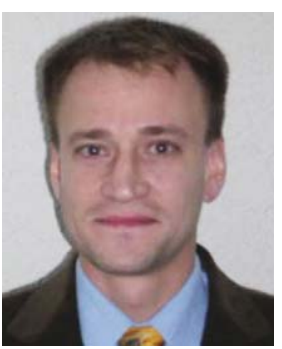

Holger Winkelmann received his Diplom degree in electrical engineering from the Fachhochschule Südwestfalen in Hagen, Germany, in 2006. Since 2006 he has been a research assistant with the University of $\mathrm{Ha}$ gen, Germany. His research interests include solar cells, micromachining, and optical sensors.

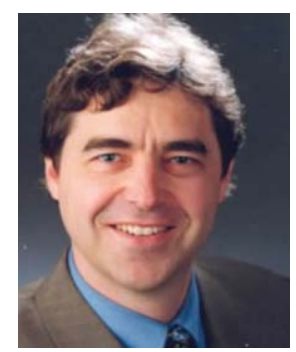

Hans Knuppertz received his Diplom degree in business administration in 1988. Until 2002 , he was a product and marketing manager for CAD and ERP software dedicated for electrotechnical companies. At the same time, he joined a distance learning program at the University of Hagen, Germany. He received his Diplom degree and his doctorate in electrical engineering in 2001 and 2008 , respectively. Dr. Knuppertz is currently a senior researcher in the optical microsystems group of the Department of Mathematics and Computer Science of the University of Hagen. 